\title{
A eficácia do treinamento auditivo formal em indivíduos com transtorno de processamento auditivo
}

\author{
Formal auditory training efficacy in individuals with auditory \\ processing disorder
}

Tatiane Eisencraft Zalcman ${ }^{1}$, Eliane Schochat ${ }^{2}$

\begin{abstract}
RESUMO
Objetivo: Verificar a eficácia de um programa de Treinamento Auditivo comparando o desempenho inicial, nos testes comportamentais, com o desempenho após o treinamento auditivo aplicado em indivíduos com Transtorno de Processamento Auditivo. Métodos: Participaram do estudo 30 sujeitos com idades entre oito e 16 anos, que passaram por uma avaliação comportamental inicial do processamento auditivo em que foram utilizados dois testes monóticos e dois dicóticos. Posteriormente foram submetidos a um programa de treinamento de auditivo durante oito semanas, a fim de reabilitar as habilidades auditivas encontradas alteradas na avaliação inicial do processamento auditivo e por fim passaram por uma nova avaliação comportamental do processamento auditivo. Resultados: Após o treinamento auditivo houve melhora em todos os testes aplicados. No teste PSI, pré-treinamento auditivo, as crianças, as crianças tinham uma média de acerto de $66,8 \%$ que passou para $86,2 \%$ após o treinamento auditivo. No teste de fala com ruído, as crianças tinham uma média de acerto de 69,3\% pré-treinamento auditivo que passou a ser 80,5\% pós-treinamento auditivo. No teste DNV, a média de acerto pré-treinamento auditivo era de 72,6\% e passou a ser $91,4 \%$. Finalmente, no teste SSW a treinamento auditivo média de acerto era de $42,2 \%$ pré-treinamento auditivo e passou a ser $88,9 \%$ pós. Conclusão: O programa de treinamento auditivo utilizado foi eficaz na reabilitação das habilidades auditivas encontradas alteradas nas crianças com Transtorno de Processamento Auditivo.
\end{abstract}

DESCRITORES: Transtornos da audição/terapia; Reabilitação de deficientes auditivos; Perda auditiva/reabilitação; Plasticidade neuronal

\section{INTRODUÇÃO}

O Transtorno de Processamento Auditivo (TPA) é um grupo complexo e heterogêneo de alterações usualmente associado a uma série de dificuldades auditivas e de aprendizado, porém havendo normalidade da audição periférica.

Estudos anteriores ${ }^{(1)}$ descreveram o TPA como um déficit em um ou mais processos auditivos centrais, sendo caracterizado por uma ou mais alterações nas habilidades de localização e lateralização sonora, discriminação e reconhecimento

Trabalho realizado no Centro de Docência e Pesquisa de Fonoaudiologia da Faculdade de Medicina da Universidade de São Paulo - USP - São Paulo (SP), Brasil, com auxílio à pesquisa da Fundação de Amparo à Pesquisa do Estado de São Paulo - FAPESP.

(1) Pós-graduanda do Programa de Pós-graduação em Ciências da Reabilitação da Faculdade de Medicina da Universidade de São Paulo - USP - São Paulo (SP), Brasil.

(2) Livre Docente, Professora do Curso de Fonoaudiologia da Faculdade de Medicina da Universidade de São Paulo - USP - São Paulo (SP), Brasil.

Endereço para correspondência: Tatiane Eisencraft Zalcman. R. Alberto Faria, 74, Alto de Pinheiros, São Paulo - SP, CEP 05459-000. E-mail: tatianeft@uol.com.br

Recebido em: 6/7/2007; Aceito em: 25/11/2007 auditivo; aspectos temporais; resolução, mascaramento, integração e ordenação temporal.

Pesquisa brasileira ${ }^{(2)}$ relata que o fato de um indivíduo apresentar audição dentro da faixa de normalidade para tons puros e teste de inteligibilidade de fala, não implica que ele esteja apto para processar e interpretar as informações auditivas expostas em situação de vida diária, levando-se em consideração todas as variáveis que podem interferir na maneira pela qual for apresentada.

Na tentativa de minimizar as habilidades auditivas alteradas encontradas no indivíduo, alguns procedimentos são propostos, entre eles o Treinamento Auditivo (TA).

O TA é um conjunto de estratégias utilizadas para desenvolver ou reabilitar as habilidades auditivas, as quais são necessárias para a compreensão da fala. O TA envolve não só a estimulação da função auditiva, como também a orientação a terapeutas, pais, educadores e a melhora do ambiente acústico ${ }^{(3)}$.

O treinamento auditivo é uma técnica amplamente utilizada na intervenção em indivíduos com distúrbio de processamento auditivo, sendo que trabalhos recentes indicam que o TA pode ter influência positiva no processamento 
temporal de crianças com dificuldades lingüísticas e de aprendizagem $^{(4-6)}$. De acordo com a literatura ${ }^{(1)}$, o TA é indicado para melhorar a função do sistema auditivo na resolução de sinais acústicos.

Três fatores ligados à plasticidade são relevantes ao Treinamento Auditivo: potencialização de longa duração (LTP Long-Term Potentiation), privação auditiva, que não é o caso dos sujeitos deste estudo e estimulação auditiva.

Quando um animal ou um ser humano é exposto repetidamente a um estímulo acústico pode aumentar o LTP e também a percepção para os estímulos repetidos. $\mathrm{O}$ treinamento auditivo e outras formas de intervenção comportamental, que promovam a estimulação auditiva, podem aumentar a atividade sináptica e através desse aumento, facilitar mudanças comportamentais $^{(7)}$.

As modificações que podem ocorrer na atividade neural devido à prática de uma habilidade, ou exposição freqüiente a um estímulo, são denominadas plasticidade neuronal ${ }^{(8)}$.

De acordo com a literatura ${ }^{(9)}$, há diversas evidências de que programas de Treinamento Auditivo (TA) podem melhorar funções auditivas elevadas, sendo tal afirmação fundamentada no fato de que o sucesso do TA é baseado na plasticidade neural.

Sendo assim, realizamos um estudo com o objetivo de verificar a eficácia de um programa de Treinamento Auditivo comparando o desempenho, nos testes comportamentais, inicial com o desempenho após o treinamento aplicado em 30 indivíduos com Transtorno de Processamento Auditivo que procuraram o Laboratório de Investigação Fonoaudiológica em Processamento Auditivo no Centro de Docência e Pesquisa em Fisioterapia, Fonoaudiologia e Terapia Ocupacional da Faculdade de Medicina da Universidade de São Paulo.

\section{MÉTODOS}

O presente estudo foi aprovado pela Comissão de Ética para Análise de Projetos de Pesquisa - CAPPesq da Diretoria Clinica do Hospital das Clinicas e da Faculdade de Medicina da Universidade de São Paulo (FMUSP), sob o Protocolo de Pesquisa $\mathrm{n}^{\circ} 1014 / 05$.

O estudo foi realizado no Centro de Docência e Pesquisa em Fisioterapia, Fonoaudiologia e Terapia Ocupacional da Faculdade de Medicina da Universidade de São Paulo.

Participaram do estudo 30 sujeitos com idades entre oito e 16 anos, sendo 12 do sexo feminino e 18 do sexo masculino. Os critérios de seleção da amostra foram: Avaliação Audiológica básica (audiometria, logoaudiometria e imitanciometria) dentro os limites da normalidade; sem quei- xas e história atuais de afecções do sistema auditivo, apresentar na avaliação do Processamento Auditivo pelo menos dois testes alterados.

Para a avaliação do PA foram utilizados dois testes monóticos e dois testes dicóticos. Dentre os testes monóticos, foi realizado o teste de fala com ruído, utilizado para a avaliação da atenção seletiva e o de fechamento auditivo. $\mathrm{O}$ outro teste monótico foi o de identificação de sentenças com mensagem competitiva (PSI em português) onde são avaliadas as habilidades auditivas de figura-fundo e atenção seletiva.

Dentre os testes dicóticos estão o não-verbal de escuta direcionada, que tem por objetivo verificar a atenção seletiva através de tarefa de separação bianual e o outro teste dicótico utilizado foi o de dissílabos alternados - SSW, que avalia a habilidade de figura-fundo para sons linguiísticos.

Após a avaliação de PA, os 30 indivíduos portadores de Transtorno de Processamento Auditivo foram submetidos a um Programa de Treinamento Auditivo baseado num procedimento eclético proposto por pesquisas anteriores ${ }^{(10,11)}$ e validado posteriormente ${ }^{(5)}$.

O Treinamento Auditivo consistiu em oito sessões, sendo uma por semana, com duração de 50 minutos cada, onde foram trabalhadas as habilidades auditivas alteradas encontradas nas avaliações dos indivíduos. Os pacientes e responsáveis também foram orientados a realizar tarefas em casa, as quais deveriam durar em média 15 minutos, devendo ser realizadas de três a quatro vezes por semana.

A dificuldade de cada tarefa do TA em cabina foi regulada para cada teste e para cada sessão com o objetivo de manter o índice de sucesso versus erro aproximado de $70 / 30 \%{ }^{(5)}$. Os procedimentos de treinamento foram agendados de forma que o mesmo tipo de tarefa raramente fosse utilizado em sessões seguidas.

\section{RESULTADOS}

Seguindo os critérios de seleção da amostra descritos anteriormente, participaram do nosso estudo 30 crianças.

A Tabela 1 demonstra a distribuição das crianças quanto ao sexo e faixa etária.

$\mathrm{Na}$ análise estatística dos dados pré e pós treinamento auditivo, utilizamos o teste Wilcoxon onde o nível de significância adotado foi de 0,05 , não foram observadas diferenças que pudessem ser consideradas estatisticamente significantes entre as orelhas direita e esquerda $(\mathrm{p}=0,85)$, sendo assim, considerou-se os valores pré e pós treinamento auditivo de ambas as orelhas, dobrando o número da amostra e aumentando, assim, a fidedignidade dos resultados (Tabelas 2 a 5 e Figura 1).

Tabela 1. Distribuição das crianças em relação ao sexo e à faixa etária

\begin{tabular}{|c|c|c|c|c|c|c|c|c|c|c|}
\hline \multirow[b]{2}{*}{ Sexo } & \multicolumn{9}{|c|}{ Faixa Etária (anos) } & \multirow[b]{2}{*}{ Total } \\
\hline & 8 & 9 & 10 & 11 & 12 & 13 & 14 & 15 & 16 & \\
\hline Feminino & 2 & 5 & 2 & 1 & 1 & 1 & 0 & 0 & 0 & 12 \\
\hline Masculino & 3 & 2 & 6 & 1 & 3 & 0 & 1 & 1 & 1 & 18 \\
\hline Total & 5 & 7 & 8 & 2 & 4 & 1 & 1 & 1 & 1 & 30 \\
\hline
\end{tabular}


Tabela 2. Comparação das avaliações pré e pós treinamento auditivo PSI

\begin{tabular}{lcc}
\hline GE $(\mathrm{n}=30)$ & \multicolumn{2}{c}{ PSI } \\
& Pré & Pós \\
\hline Média & $66,8 \%$ & $86,2 \%$ \\
Mediana & $70,0 \%$ & $90,0 \%$ \\
Desvio Padrão & $18,0 \%$ & $12,9 \%$ \\
Quartil 1 & $50,0 \%$ & $80,0 \%$ \\
Quartil 3 & $80,0 \%$ & $100,0 \%$ \\
Tamanho & 60 & 60 \\
IC & $4,6 \%$ & $3,3 \%$ \\
\hline
\end{tabular}

p-valor $<0,001^{*}$

Legenda: PSI - Teste de Identificação de Sentenças com Mensagem Competitiva; IC - Intervalo de Confiança.

Tabela 3. Comparação das avaliações pré e pós treinamento auditivo Fala com Ruído

\begin{tabular}{lcc}
\hline GE $(\mathrm{n}=30)$ & \multicolumn{2}{c}{ Fala c/ Ruído } \\
& Pré & Pós \\
\hline Média & $69,3 \%$ & $80,5 \%$ \\
Mediana & $68,0 \%$ & $80,0 \%$ \\
Desvio Padrão & $10,9 \%$ & $7,3 \%$ \\
Quartil 1 & $64,0 \%$ & $76,0 \%$ \\
Quartil 3 & $76,0 \%$ & $84,0 \%$ \\
Tamanho & 60 & 60 \\
IC & $2,8 \%$ & $1,9 \%$ \\
\hline
\end{tabular}

p-valor $<0,001^{*}$

Legenda: IC - Intervalo de Confiança.

Tabela 4. Comparação das avaliações pré e pós treinamento auditivoDicótico Não Verbal

\begin{tabular}{lcc}
\hline GE $(\mathrm{n}=30) \mathrm{I}$ & Pré & DNV \\
\hline Média & 8,72 & Pós \\
Mediana & 9 & 10,97 \\
Desvio Padrão & 2,53 & 12 \\
Quartil 1 & 7 & 1,76 \\
Quartil 3 & 11 & 11 \\
Tamanho & 60 & 12 \\
IC & 0,64 & 60 \\
p-valor & & 0,44 \\
\hline
\end{tabular}

Legenda: DNV- Dicótico não verbal; IC - Intervalo de Confiança;

Tabela 5. Comparação das avaliações pré e pós treinamento auditivo SSW

\begin{tabular}{lcc}
\hline GE $(\mathrm{n}=30)$ & \multicolumn{2}{c}{ SSW } \\
& Pré & Pós \\
\hline Média & $72,2 \%$ & $88,9 \%$ \\
Mediana & $72,5 \%$ & $92,5 \%$ \\
Desvio Padrão & $11,9 \%$ & $11,0 \%$ \\
Quartil 1 & $65,0 \%$ & $87,5 \%$ \\
Quartil 3 & $82,5 \%$ & $95,0 \%$ \\
Tamanho & 60 & 60 \\
IC & 0,64 & $2,8 \%$ \\
\hline
\end{tabular}

p-valor

$<0,001^{*}$

Legenda: SSW - Teste de dissílabos alternados; IC - Intervalo de Confiança

Rev Soc Bras Fonoaudiol. 2007;12(4):310-4

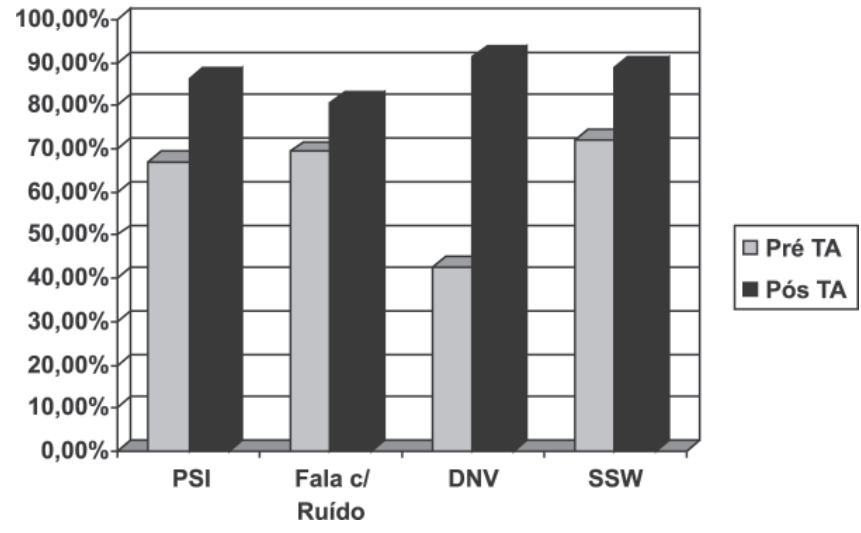

Figura 1. Comparação entre a avaliação comportamental do Processamento Auditivo Pré e Pós Treinamento auditivo.

\section{DISCUSSÃO}

Os denominados transtornos de Processamento Auditivo, caracterizados como disfunções especificamente auditivas, constituem uma categoria ampla e diversificada dentro dos distúrbios da audição, que podem estar relacionados a lesões do Sistema Nervoso Auditivo ou a outros processos de caráter funcional ${ }^{(12)}$.

Estudos anteriores ${ }^{(13)}$ destacaram que as principais causas dos transtornos de processamento auditivo poderiam ser devido a alterações das condições neurológicas, atraso de maturação do sistema nervoso central e coexistência com outras disfunções do desenvolvimento.

$\mathrm{Na}$ literatura ${ }^{(6)}$, foram encontrados relatos de que o TA era utilizado em indivíduos que apresentavam deficiência auditiva de grau profundo, a fim de otimizar o uso de sua audição residual. Com o tempo, o TA passou a ser utilizado em indivíduos com perdas auditivas menores para ajudar na adaptação das próteses auditivas e melhorar o uso da amplificação e esses estudos mostraram que o TA não melhora o limiar auditivo, porém melhora a percepção de sinais acústicos mais complexos como a fala.

Diferentes autores ${ }^{(1,9,14-19)}$ citaram as características gerais de um programa de treinamento auditivo eficaz e apesar de algumas diferenças, todos são unânimes em afirmar que o mesmo precisa ser intensivo, conter atividades desafiantes ao sistema auditivo e suficientemente interessante de modo a manter a motivação do paciente, evitando sua frustração.

Podemos verificar que no grupo estudado a média de acertos após o TA em todos os testes utilizados na avaliação inicial e que foram repetidos na avaliação final (após o TA) apresentaram uma diferença estatisticamente significante, sendo melhor após o TA.

Essa melhora após o TA pode ser relacionada com a idéia defendida por outros autores ${ }^{(20)}$, em que a melhora das habilidades auditivas provavelmente surgiu como resposta a influência ambiental previamente determinada e modificada de maneira desejada, que levou uma mudança neural associada. Ou seja, a influência ambiental, mais especificamente o TA foi capaz de estimular as estruturas neurais relacionadas ao desempenho das habilidades auditivas treinadas, benefi- 
ciando assim o indivíduo.

Em um estudo realizado anteriormente ${ }^{(21)}$, os autores realizaram um treinamento auditivo nos mesmos moldes realizado em nosso estudo, ou seja, trabalharam as habilidades auditivas alteradas encontradas na avaliação inicial do processamento auditivo, durante oito semanas com sessões de aproximadamente 50 minutos, uma vez por semana. A faixa etária dos sujeitos foi a mesma utilizada em nosso estudo, ou seja, entre oito e 16 anos. O resultado da avaliação comportamental após o treinamento auditivo, verificou uma melhora nos testes comportamentais com um aumento estatisticamente significante em todos os testes utilizados (PSI, Fala com ruído, DNV e SSW) o que corrobora os achados do nosso estudo

Em outro estudo ${ }^{(22)}$, os autores compararam o desempenho comportamental em atividades que envolvem audição e linguagem de escolares com distúrbio de processamento auditivo, antes e após treinamento auditivo. Neste estudo, diferentemente do nosso, as crianças que foram caracterizadas como portadoras do DPA foram encaminhadas para uma avaliação fonoaudiológica completa e posterior estimulação auditiva durante nove semanas, que envolveram os seguintes aspectos: habilidades de memória de coisas aprendidas anteriormente, memória imediata, treinamento de tônus e mobilidade dos órgãos fonoarticulatórios, orientação têmporo-espacial, voz, ritmo, seqüêncialização, leitura e escrita, compreensão de fala no ruído. Após as nove semanas de treinamento, as crianças foram reavaliadas e das 21 crianças reavaliadas, 15 apresentaram resultados dentro da normalidade e seis mantiveram algum tipo de alteração. Em nosso estudo realizamos um treinamento auditivo em cabine acústica, durante oito semanas, uma vez por semana, que visou trabalhar as habilidades auditivas alteradas encontradas na avaliação do processamento auditivo. Apesar, de formas diferentes de treinamento auditivo, em ambos os estudos as crianças com distúrbio no processamento auditivo obtiveram melhor desempenho nos testes comportamentais que avaliam o processamento auditivo após o treinamento auditivo, portanto tiveram uma melho- ra nas habilidades auditivas treinadas.

Em nosso estudo, dos 30 sujeitos (100\%) que apresentavam pelo menos dois testes alterados na avaliação comportamental inicial do Processamento Auditivo, apenas 11 $(36,66)$ continuaram alterados após serem submetidos ao programa de treinamento auditivo, sendo que 19 (63,33\%) sujeitos apresentaram normalidade em todos os testes aplicados após o treinamento.

Plasticidade neural é definida ${ }^{(20)}$ como uma mudança em células nervosas que ocorrem de acordo com as influências ambientais, e se estas influências podem ser controladas e modeladas da maneira desejada, o comportamento relacionado à plasticidade pode ser previsível.

Segundo estudos anteriores ${ }^{(9)}$, podem ocorrer três tipos de plasticidade no sistema auditivo: plasticidade desenvolvimental; plasticidade compensatória, resultante de uma lesão ocorrida no sistema auditivo e a plasticidade relacionada à aprendizagem. Observamos, portanto, que a plasticidade ocorrida neste estudo foi à relacionada com a aprendizagem, uma vez que as crianças foram submetidas a um programa de treinamento das habilidades auditivas que se encontravam alteradas.

Assim, através de um programa de treinamento auditivo, surgiram evidências que sugerem que o sistema auditivo central de crianças pode modificar-se com o auxílio do treinamento auditivo revelando a plasticidade do sistema nervoso auditivo central.

\section{CONCLUSÃO}

A partir da análise dos resultados obtidos no presente estudo, podemos concluir que o programa de treinamento auditivo utilizado foi eficaz na reabilitação das habilidades auditivas encontradas alteradas nas crianças com Transtorno de Processamento Auditivo, visto que 63,3\% das crianças apresentaram o resultado da avaliação de Processamento Auditivo dentro dos limites de normalidade após o programa de Treinamento Auditivo.

\begin{abstract}
Purpose: To assess the effectiveness of the Auditory Training comparing the performance in the behavioral tests before and after auditory training in individuals with Auditory Processing Disorders. Methods: Thirty individuals with ages ranging from eight to 16 years were submitted to an auditory processing evaluation, which consisted of two monotic and two dichotic tests. After that, the individuals were enrolled in an auditory training program composed by 8 sessions, to rehabilitate the auditory abilities and afterwards were submitted to a re-evaluation of the auditory processing. Results: After the Auditory Training we observed an improvement in the results of all behavioral tests. On the PSI test the performance before auditory training was $66,8 \%$ and after, $86,2 \%$. On the speech in noise test, before auditory training, the results were $69,3 \%$ and after auditory training, $80,5 \%$. On the Nonverbal Dichotic test, before auditory training, the performance of the individuals was $72,6 \%$ and after training the performance was $91,4 \%$. Finally, in the SSW test before auditory training, the results were $42,2 \%$ and after auditory training, $88,9 \%$. Conclusion: Auditory training program was considered effective for rehabilitation of auditory abilities in individuals with auditory processing disorders.
\end{abstract}

KEYWORDS: Hearing disorders/therapy; Rehabilitation of hearing impaired; Hearing loss/rehabilitation; Neuronal plasticity 


\section{REFERENCIAS}

1. Chermak GD, Musiek FE. Auditory training: principles and approaches for remediating and managing auditory processing disorders. Semin Hear. 2002;23(4):297-308.

2. Pereira LD, Schochat E. Processamento auditivo central: manual de avaliação. São Paulo: Lovise; 1997. 231p.

3. Bamford J. Auditory train. What is it, what is it supposed to do, and does it do it? Br J Audiol. 1981;15(2):75-8.

4. Tallal P, Miller SL, Bedi G, Byma G, Wang X, Nagarajan SS, et al. Language comprehension in language-learning impaired children improved with acoustically modified speech. Science. 1996;271(5245):81-84. Comment in: Science. 1996;271(5245):27-8.

5. Musiek FE, Schochat E. Auditory training and central auditory processing disorders: a case study. Semin Hear. 1998;19(4):357-66.

6. Musiek FE. Central auditory tests. Scand Audiol Suppl. 1999;51:33-46.

7. Chermak GD, Musiek FE, editors. Handbook of (Central) auditory processing disorder . Comprehensive intervention. San Diego: Plural Publishing; 2006. v.2.

8. Grafman J. Conceptualizing functional neuroplasticity. J Commun Disord. 2000;33(4):346-55; quiz 355-6.

9. Musiek FE, Shinn J, Hare C. Plasticity, auditory training, and auditory processing disorders. Semin Hear. 2002;23(4):263-76.

10. Chermak GD, Musiek FE. Managing central auditory processing disorders in children and youth. Am J Audiol. 1992;1(3):61-5.

11. Musiek FE, Chermak GD. Three commonly asked questions about central auditory processing disorders: management. Am J Audiol. 1995;4(1):15-8.

12. Jerger J, Musiek F. Report of the Consensus Conference on the Diagnosis of Auditory Processing Disorders in School-Aged Children. J Am Acad Audiol. 2000;11(9):467-74.
13. Bamiou DE, Musiek FE, Luxon LM. Aetiology and clinical presentations of auditory processing disorders - a review. Arch Dis Child. 2001;85(5):361-5.

14. Bode DL, Oyer HJ. Auditory training and speech discrimination. J Speech Hear Res. 1970;13(4):839-55.

15. Merzenich MM, Jenkins WM, Johnston P, Schreiner C, Miller SL, Tallal P. Temporal processing deficits of language-learning impaired children ameliorated by training. Science.1996;271(5245):77-81. Comment in: Science. 1996;271(5245):27-8.

16. Chermak GD, Musiek FE, Craig CH. Fundamental concepts and considerations for management. In: Chermak GD, Musiek FE, Craig CH. Central auditory processing disorders: new perspectives. San Diego: Singular Publishing Group; 1998. p. 151-83.

17. Schochat E. Insights for management of processing disorders. Hear J. 2004;57(10):58.

18. Sweetow RW, Henderson-Sabes J. The case for LACE: listening and auditory communication enhancement training. Hear J. 2004;57(3):328.

19. Sweetow RW. Training the adult brain to hear. Hear J. 2005;58(6):10-6.

20. Musiek FE, Berge B. A neuroscience view of auditory training/ stimulation and central auditory processing disorders. In: Masters M, Stecker N, Katz J, editors. Central auditory processing disorders: mostly management. Boston: Allyn \& Bacon; 1998. cap 2. p 15-32.

21. Schochat E, Musiek F, Alonso R. The effects of auditory training on the Middle Latency Response (MLR) in children with APD. Journal of the academy of audiology. 2007 (prelo).

22. Beck CS, Calichman F, Gandra LPF, Machado AH, Pereira LD. Estimulação do processamento aditivo central em escolares de 7 a 10 anos de idade. Pró-Fono. 1996;8(2):45-50. 